\title{
Stem cells: finding the right path
}

\author{
Nathan Blow
}

\section{With increasing numbers of well-characterized stem cell lines and improved culture and differentiation technologies, more scientists are testing the waters of stem cell research.}

Over the past year, Jessie $\mathrm{Ni}$, director of the stem cell department at R\&D Systems in Minneapolis, has been attending a variety of scientific conferences, giving tutorial presentations on stem cell culture and use. Although the members of her audience have often had different reasons for potentially using stem cells, their interest has been undeniable. "Everybody will come up after the presentation and ask 'how I can do this' or 'how I can do that'” says Ni.

This high level of interest in stem cells is leading commercial developers to address the needs of researchers entering the field. To this end, companies such as R\&D Systems, Thermo Fisher Scientific in Waltham, Massachusetts, USA, and Millipore in Billerica, Massachusetts, USA, now offer adult and embryonic stem cell (ESC) lines along with optimized media and differentiation reagents, all of which have been quality-controlled and validated, taking away some of the initial challenge for novice stem cell researchers.

At the same time, major initiatives within the stem cell community are focused on evaluating the robustness of the many different culture systems currently in use. Martin Pera, director of the Institute for Stem Cell and Regenerative Medicine at the University of Southern California, is a member of the International Stem Cell Initiative (ISCI), a worldwide effort to establish basic criteria and techniques for using human ESCs. "These systems are now undergoing evaluation by the ISCI as part of a multilab comparison for their ability to support short- and long-term ES cell growth with normal karyotypes," says Pera about the current ISCI study, which he expects will be completed and released in 2009 (Box 1). Meanwhile, in England, Stem Cells for
Safer Medicines, a recently founded publicprivate partnership, is also evaluating various culture systems in an effort to establish open standards and protocols, and evaluate the utility of differentiated cells in early drug safety screening, says Philip Wright, chief executive of the company and director of science and technology at the Association of the British Pharmaceutical Industry.

\section{Defining moments}

Culturing stem cells is both a science and an art, and it can be a challenge to keep these cells stable and healthy. ESCs are pluripotent - they have the potential to generate all adult and embryonic cell types - whereas adult stem cells are multipotent, that is, capable of generating only specific cell types. Specific chemical or envi- ronmental cues direct stem cells to differentiate into new cell types. These differentiation signals vary between different types of stem cells and are not fully understood for most cell lineages, making the composition of the different media used for propagating as well as differentiating these cells critical. Given this, it should come as no surprise that everyone in the field is moving toward the use of defined culture media.

"In general, dealing with undefined media is a problem in both basic and applied research," notes Sheng Ding, a chemist at The Scripps Research Institute in La Jolla, California. He says that culture systems that rely on serum or use media with unknown composition tend to be less reproducible and are often prone to batch-to-batch variation. According to Ding, scientists using

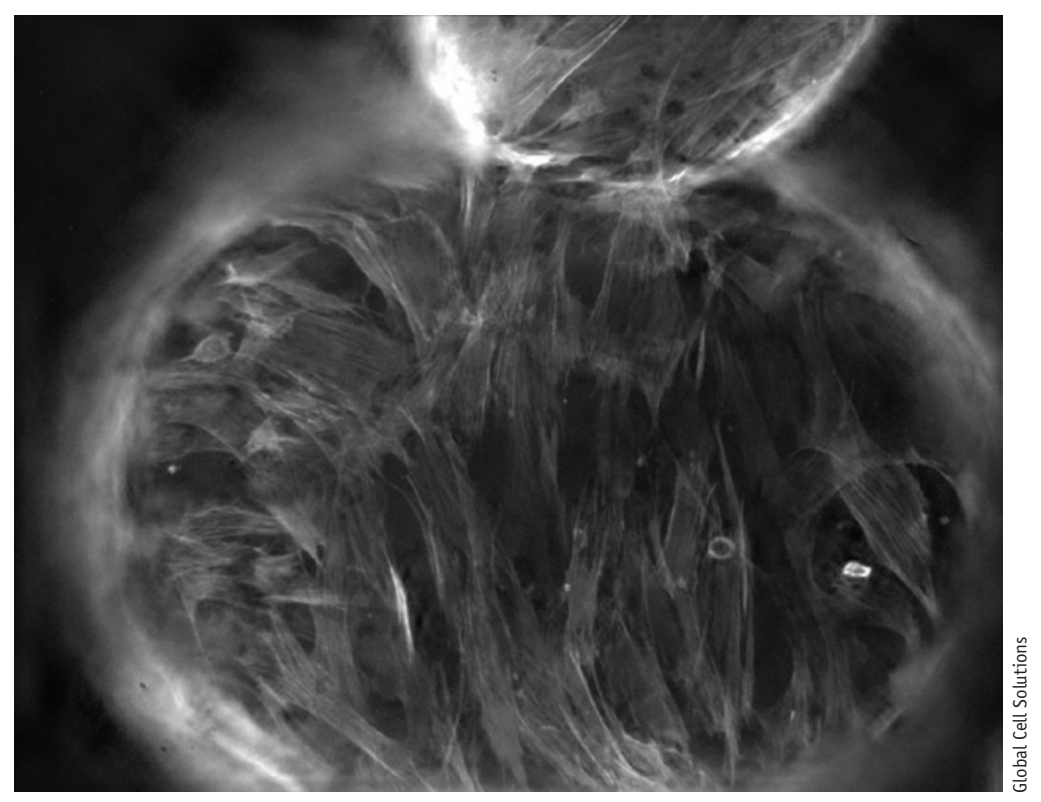

Microcarriers can provide effective surfaces for both the growth and differentiation of stem cells. 


\section{BOX 1 CULTURAL CHANGES}

In 2005, cytogeneticists Lorraine Meisner and Julie Johnson were working in a clinical laboratory at the University of Wisconsin, Madison. Along with the clinical samples they were processing, Meisner and Johnson were starting to see a wave of samples coming in from stem cell researchers for chromosome analysis. "But they were always put at the low end of the totem pole; these were not high priority samples," says Johnson.

As the number of research samples increased, it was taking longer and longer to get the results out of the lab. This was when Meisner and Johnson decided to try something different. "We thought that we could do this on our own and provide faster service to the stem cell community," recalls Johnson. So they decided to form a company dedicated to cytogenetics service and thus Cell Line Genetics was born.

One of the goals of the company, which was founded in 2006 , is to provide rapid cytogenetic services to detect anomalies in both research and clinical stem cell lines. The company has now performed cytogenetic analyses on more than 1,000 human and mouse stem cell lines looking for chromosomal abnormalities. “We did 725 human ESC lines of which 233 had chromosomal abnormalities, and 275 mouse ESC lines of which $69 \%$ were abnormal," notes Meisner. Most surprising of all might be the trends that Meisner and Johnson have seen in their analyses, indicating that these are not completely random chromosomal changes.

"The interesting thing is that no matter the medium or the country of origin, ESCs still select specific chromosomal aberrations that are adaptive for those cells," says Meisner. This would mean that although the chromosomal aberrations arise at random, it is the ones that are adaptive for growth in culture that remain and are propagated.

"We know that these abnormalities occur, and it appears that certain types of culture conditions tend to favor their appearance," notes Martin Pera, adding that it is not as yet clear what these favorable conditions might be. But Pera does think that large-scale efforts like the International Stem Cell Initiative (ISCI), which is looking at the effects of culture conditions on different stem cell lines, could begin to provide insights into the issue.

In the meantime, Meisner and Johnson say that some of the aberrations they have identified demonstrate the need for more comprehensive cytogenetic analysis. One such aberration, which has been detected in both human and mouse ESC lines, is pseudodiploidy, where 46 chromosomes are present, but instead of two copies of chromosome 17, for example, the cell has three copies and is missing a copy of another chromosome. "This is something we are trying to eliminate in the field. Some researchers think they can do a chromosome prep and count, but the high rates of pseudo-diploidy we are finding indicate chromosome counts are not enough to detect certain aberrations," says Johnson.

Pera has another concern when it comes to these genetic changes in stem cells. "We still do not understand the magnitude of the potential problem of smaller genetic changes that are not visible at the microscopic level." This is something else the ISCI members are investigating, as the group collects a wide range of cell lines at various passage numbers and performs both karyotype and single nucleotide polymorphism analyses to look at genetic changes in cultured stem cells over time.

In the future, Cell Line Genetics hopes to provide technology to enable scientists themselves to assess the integrity of their stem cells with regard to the most commonly identified chromosomal abnormalities. But, for now, Meisner and Johnson have their hands full, as that wave of research samples shows no signs of declining. these systems to hunt for specific molecules or genes involved in stem cell differentiation can feel "like they are walking in the dark," as interactions with unknown factors can potentially contribute to the differentiated cellular phenotype.

More and more companies are now supplying serum-free media for culturing stem cells. Invitrogen, located in Carlsbad, California, USA, offers a medium called StemPro hESC SFM, which supports the growth of over 40 human ESC lines from around the world in a serum-free environment, and Millipore supplies HEScGRO, which is another serum-free medium that has been shown to work with a variety of human ESC lines as well as ESGRO Complete Clonal Grade Medium for mouse ESC culture.

When it comes to differentiating stem cells in culture, however, the number of lineages for which defined conditions are now available is limited. There are only a couple of well-defined chemical conditions for neural stem cell differentiation at the moment, and work toward the same goal for other lineages is still ongoing.

Although medium composition is one challenge, another issue for researchers working with human ESCs arises from the use of feeder cells. Mouse embryonic fibroblast cells (MEFs) have been used in the past to sustain human ESCs in culture. But MEFs can also present problems for downstream applications resulting from the presence of xenogeneic molecules from the different species_potentially limiting their clinical use in the future. It is thought that MEFs provide essential growth factors to ESCs, an idea that led scientists to hunt for these essential factors. In 2006, researchers at the WiCell Research Institute in Madison, Wisconsin, USA reported the development of a new medium formulation, called TeSR1, which was supplemented with protein components that were either recombinant or purified from human material, allowing culture of human ESCs without using MEFs. The latest generation of TeSR1 medium, now called

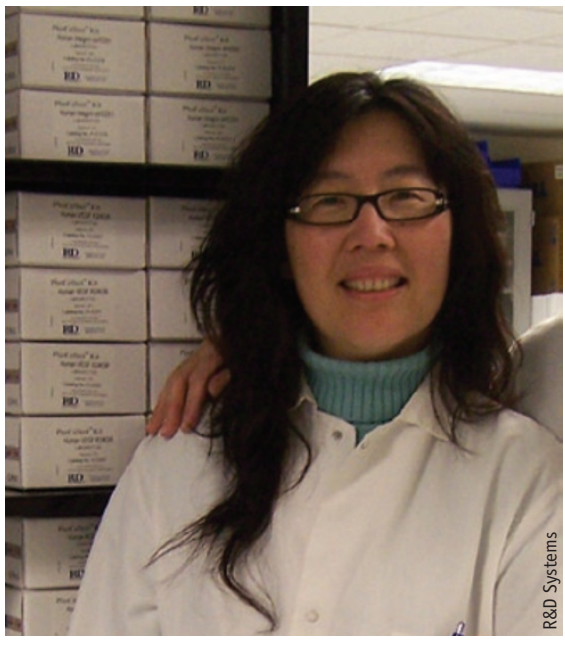

Jessie Ni from R\&D Systems has been seeing a rise in interest among scientists in using stem cells. 


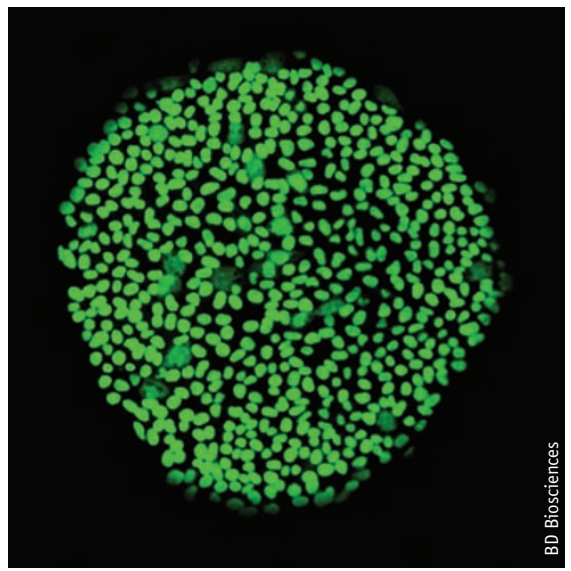

Staining of undifferentiated human ESCs cultured on BD Matrigel in mTeSR-1 medium.

mTeSR-1, is being marketed by StemCell Technologies in Vancouver, Canada, in collaboration with BD Biosciences in San Jose, California, USA, for use in some applications with BD Biosciences' Matrigel scaffold, the matrix used in the initial experiments with TeSR1 medium.

Matrigel is a solubilized protein preparation extracted from mouse sarcomas that can be coated onto tissue-culture dishes as a two-dimensional scaffold for long-term culture of human ESCs. In its current format, Matrigel can present similar issues to the use of serum when culturing stem cells, although researchers at BD Biosciences are working now to develop a more defined version of the scaffold.

Like media, defined scaffolds are becoming increasingly commonplace, not only for culturing adult and embryonic stem cells but also for inducing differentiation through the physical interactions of cells with other cells and the surrounding environment.

Global Cell Solutions in Charlottesville, Virginia, USA has developed an alginatebased microcarrier technology termed global eukaryotic microcarrier (GEM) for culturing cells in three dimensions. The GEM core is composed of a biopolymer called alginate with embedded magnetic particles, and its surface can be coated with covalently bound gelatin or other substrates, which can be functionalized with small molecules to create a surface for growth or differentiation. "Our ability to put different coatings on the surface has really piqued the interest of stem cell researchers," notes Uday Gupta, president and chief executive officer of Global Cell Solutions.

"We are able to manipulate the entire system as a pipettable substrate," says Brad
Justice, director of research at the company. Justice also notes that, on account of the magnetic particles lodged in the alginate, the GEM microcarriers can be immobilized when changing media or performing separations.

Other three-dimensional matrix systems are available now as well. Novamatrix (FMC Biopolymer) located in Sandvika, Norway uses alginate beads and foams to look at differentiation in three dimensions compared to two, and Invitrogen also offers an alginate-based matrix shaped like an egg crate, which allows the formation of spheroidal aggregate structures in a controlled environment. Polystyrene, the basis of tissue culture dishes, is even being advanced for use in the three-dimensional culture of stem cells by a company called Reinnervate in Durham, UK.

\section{Small-molecule bailout}

Ding's group at The Scripps Research Institute is searching for new small molecules to improve culture and differentiation of stem cells. This approach actually has a long history: the naturally occurring metabolite retinoic acid has been used for more than 20 years in neural cell differentiation. But Ding is taking the concept to the next level, using high-throughput screening of large chemical libraries of biologically active compounds to rapidly identify useful molecules.

When it comes to finding compounds that drive a cell to a particular lineage, he acknowledges the task is particularly challenging. In most cases, the underlying molecular mechanisms of differentiation are not at all clear, making hypothesis-driven experiments impractical. But this is where Ding and others think discovery-based high-throughput approaches have the edge: they can be used to identify small molecules without relying on prior knowledge.

Ding says his group often screens for small molecules at multiple steps along a given differentiation pathway. "One molecule may not generate the perfect phenotype, but it will have the ability to impact the process, even though it may not be sufficient for 100\% phenotypic modulation," he explains. And by then combining the resulting 'hits', Ding hopes that small molecules could drive differentiation from the primitive cell to a completely differentiated cell type.

Small molecules are also starting to be used in the development of induced pluripotent stem (iPS) cell techniques. "The big thing now is getting to iPS cells without 
putting in potentially carcinogenic genes,' says Joydeep Goswami, vice president of stem cell research at Invitrogen. Goswami's group is working with researchers on new transfection technologies and smallmolecule approaches to generate iPS cells without leaving the 'footprint' caused by current virus-based approaches. For his part, Ding has been identifying small-molecule alternatives for generating iPS cells. Members of his lab, along with others, have identified small molecules that can replace some of the crucial transcription factors and improve reprogramming efficiency.

\section{Letting cells tell the tale}

Determining whether an ESC or an iPS cell is pluripotent or whether it has undergone differentiation in culture may get easier thanks to the work of several researchers. Goswami and his colleagues at Invitrogen have spent the past couple of years developing several engineered stem cell lines, along with a gene-targeting system, to allow researchers to monitor the state of their human ESCs.

"The core of our technology is that we were able to put a target locus at a specific point in the chromosome of the embryonic stem cell," explains Goswami. The researchers could then use this target locus, or 'hot spot', in the ESC chromosome to insert and express markers of pluripotency or other genes of interest.

Goswami explains that finding 'hot spots' with the desired properties was far from trivial. These chromosomal locations not only had to have high transfer efficiency and allow stable integration, but, just as critically, they could not be transcriptionally silenced upon ESC differentiation. To test transcriptional activity, Goswami's team first performed bioinformatics analyses to determine whether a locus of interest was likely to be active in most cell types of the

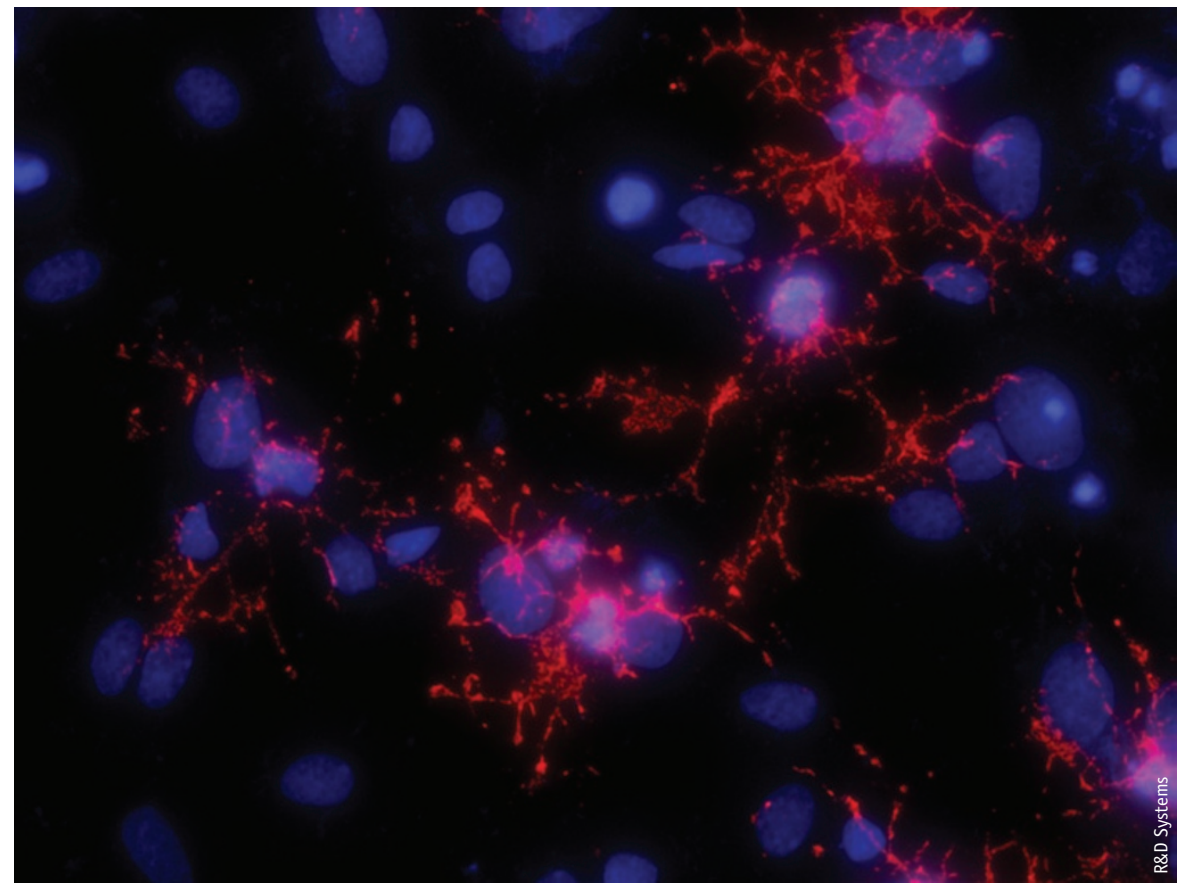

Oligodendrocytes differentiated from neural stem cells

body. Then they performed experiments looking at embryoid body formation to determine whether all three germline lineages fluoresced when fluorescent-protein marker genes were inserted into the locus, as well as by directed differentiation to ensure that the locus was active in mesenchymal and neural lineages, among other lineages. With these criteria, Goswami says they found three appropriate 'hot spots' and have developed three different engineered ESC lines thus far.

"We have just released a target line so that people can tinker around and drop their own genes into the locus and see what happens," says Goswami. The other cell lines, which are on the way, already have marker genes inserted into the target locus. For example, in one cell line, GFP is driven by the POU5F1 promoter, also known as Oct4, which turns off when ESCs differentiate, and this construct therefore acts as a marker for pluripotency. In another cell line, GFP is under the control of the constitutively active EEF1A1, also known as EF1 $\alpha$, promoter, which can be used to identify and localize engineered cells. Goswami says he is now examining neural lineages and creating engineered stem cells with promoters that turn on only when cells reach a particular neural lineage.

Other methods that can be used to monitor stem cells include a stem-cell potency assay from Applied Biosystems in Foster City, California, USA, based on their TaqMan system, which allows researchers to characterize both human and mouse ESCs based on the expression patterns of a set of 92 test genes. In addition, researchers at R\&D Systems
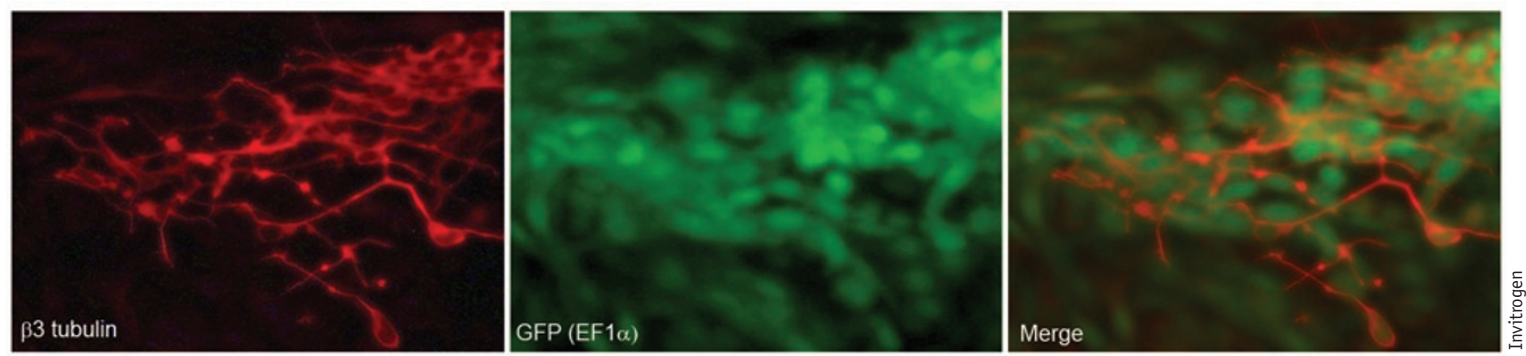

Engineered human ESCS, expressing a green fluorescent protein under the control of a constitutive promoter (EEF1A1), which had been differentiated to neurons. Neuronal axons are identified using an antibody to $\beta 3$ tubulin labeled with AlexaFluor 594 and appear red. Cell bodies expressing GFP appear green. 


\section{TECHNOLOGY FEATURE}
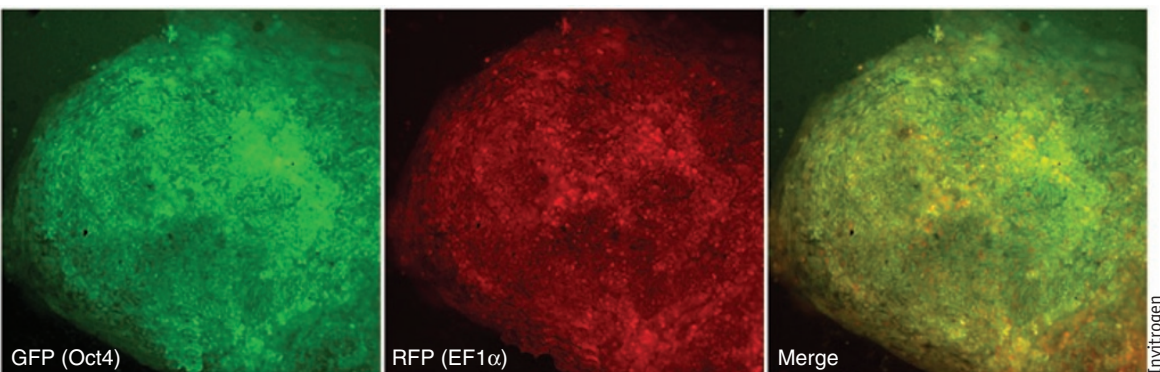

Engineered human ESCs in pluripotent state that will fluoresce at both green and red wavelengths while in the pluripotent state but rapidly lose their green fluorescence and maintain red as the cell begin to differentiate.

have been developing both antibodyand transcription-based approaches to monitor the pluripotency state of ESCs. For the former approach, the company supplies antibodies that recognize the standard pluripotency markers, including SOX2 and OCT3/4, and which can be used to monitor the 'stemness' of ESCs or iPS cells. In the latter approach, they are taking a further step toward defining the pluripotent state with the development of chromatin immunoprecipitation kits for SOX2, OCT3/4 and NANOG to identify genes regulated by these transcription factors. Ni from R\&D Systems adds that they are now advancing their chromatin immunoprecipitation assay to look not only at genes regulated by the individual transcription factors but also those regulated by complexes of these factors.

With the ability to more accurately monitor the state of ESCs developing alongside new culture and differentiation approaches, Martin Pera says the fact that more and more researchers are now exploring stem cells should not come as any shock. "I think despite the barriers the field has worked hard to improve and disseminate the technology, and that is showing up in terms of the numbers and caliber of the people now involved in stem cell research."

Nathan Blow is the technology editor for Nature and Nature Methods

(n.blow@boston.nature.com). 


\section{SUPPLIERS GUIDE: COMPANIES OFFERING STEM CELL MEDIA,}

REAGENTS AND ACCESSORIES

Company

Aastrom Biosciences

Advanced Cell Technology

Amaxa

Applied Biosystems

Beckman Coulter

BD Biosciences

Bioflow Technology

BioProcessors Corp.

Bio-Rad Laboratories

Biotec

Carl Zeiss

Cell Line Genetics

Cellartis

Cellular Engineering Technologies

EMD

ES Cell International

EUGENEX Biotechnologies

Geron

Global Cell Solutions

Glycosan BioSystems Inc.

Integra Biosciences

Invitrogen

Irvine Scientific

Leica Microsystems

Lonza

Merck

Millipore

Molecular Machines \& Industries

New England Biolabs

Nikon Instruments

Novamatrix

Novocell

Olympus America

PAA

Perkin Elmer

Princeton Instruments

PromoCell

Thermo Fisher Scientific

Reinnervate

R\&D Systems

Stemagen

StemCell Services

Stem Cells

Stem Cell Innovations

Stem Cell Technologies

Zen-Bio
Product

http://www.aastrom.com/

http://www.advancedcell.com/

http://www.amaxa.com/

http://www.appliedbiosystems.com/

http://www.beckmancoulter.com/

http://www.bd.com/

http://www.bioflowtech.com/

http://www.bioprocessors.com/

http://www.bio-rad.com/

http://www.miltenyibiotec.com/

http://www.zeiss.com/

http://www.clgenetics.com/

http://www.cellartis.com/

http://www.celleng-tech.com/

http://www.emdbiosciences.com/

http://www.escellinternational.com/

http://www.eugenex.com/

http://www.geron.com/

http://www.globalcellsolutions.com/

http://www.glycosan.com/

http://www.integra-biosciences.com/

http://www.invitrogen.com/

http://www.irvinesci.com/

http://www.leica-microsystems.com/

http://www.lonza.com/

http://www.merck.de/

http://www.millipore.com/

http://www.molecular-machines.com/ http://www.neb.com/

http://www.nikoninstruments.com/ https://www.novamatrix.biz/

http://www.novocell.com/

http://www.olympusamerica.com/

http://www.paa.com/

http://las.perkinelmer.com/

http://www.princetoninstruments.com/ http://www.promocell.com/ http://www.thermofisher.com/ http://www.reinnervate.com/ http://www.rndsystems.com/ http://www.stemagen.com/ http://www.stemcell-services.com/ http://www.stemcellsinc.com/ http://www.stemcellinnovations.com/ http://www.stemcell.com/ http://www.zen-bio.com/ 